\title{
Expression of microRNA-150 targeting vascular endothelial growth factor-A is downregulated under hypoxia during liver regeneration
}

\author{
ZHAO-YAN YU ${ }^{1,2}$, YAN-NAN BAI ${ }^{1}$, LI-XI LUO ${ }^{1}$, HONG WU $^{1}$ and YONG ZENG ${ }^{1}$ \\ ${ }^{1}$ Department of Hepato-Biliary-Pancreatic Surgery, West China Hospital, Sichuan University, Chengdu, Sichuan 610041; \\ ${ }^{2}$ Department of General Surgery, The People's Hospital of Guizhou Province, Guiyang, Guizhou 550002, P.R. China
}

Received February 4, 2013; Accepted May 3, 2013

DOI: $10.3892 / \mathrm{mmr} .2013 .1493$

\begin{abstract}
Vascular endothelial growth factor(VEGF) is a factor that stimulates the proliferation of sinusoidal endothelial cells and hepatocytes during liver regeneration (LR). The present study aimed to screen and validate a microRNA (miRNA) that targets VEGF-A with relative specificity and to elucidate the potential association between hypoxia-inducible factor- $1 \alpha$ (HIF-1 $\alpha$ ) and miRNA expression in the early phase of LR. Changes in the expression of miRNAs, which were predicted to target VEGF-A using online databases, were detected at 12, 24 and $48 \mathrm{~h}$ following a $70 \%$ partial hepatectomy (PHx) using quantitative PCR (qPCR). An inhibitor of the most downregulated miRNA was transfected into the primary hepatocytes in order to observe changes in the expression of the VEGF-A gene. The expression of HIF- $1 \alpha$ protein in the regenerating liver was investigated using western blot analysis. The expression levels of HIF- $1 \alpha$ mRNA (messenger RNA), the selected miRNA and VEGF-A mRNA in an anoxic model of hepatocytes was examined with qPCR. Of seven putative miRNAs, the expression of miR-150 exhibited the sharpest downregulation from 12-48 h. The micrOFF ${ }^{\mathrm{TM}}$ miR-150 inhibitor significantly elevated the expression levels of VEGF-A mRNA and protein $48 \mathrm{~h}$ after transfection. Thus, VEGF-A may be a downstream target of miR-150 during LR. Furthermore, HIF-1 $\alpha$ protein expression increased to its highest level $24 \mathrm{~h}$ following PHx. miR-150 expression was inhibited and the expression of VEGF-A mRNA increased accordingly in the hypoxia-induced hepatocytes. Our results suggest that miR-150 expression is subject to negative regulation by HIF-1 $\alpha$.
\end{abstract}

Correspondence to: Professor Yong Zeng, Department of Hepato-Biliary-Pancreatic Surgery, West China Hospital, Sichuan University, 37 Wainan Guoxuexiang, Chengdu, Sichuan 610041, P.R. China

E-mail: zengyong@medmail.com.cn

Key words: microRNA-150, hypoxia-inducible factor-1 $\alpha$, vascular endothelial growth factor, liver regeneration

\section{Introduction}

The liver has the ability to regenerate following injury, allowing it to rapidly and efficiently restore lost mass without jeopardizing the viability of the entire organism (1). The phenomenon of liver regeneration (LR) may be induced by various methods, including liver damage by hepatotoxic chemicals (2), the rodent model of $70 \%$ partial hepatectomy (PHx) (3) and small-for-size liver transplantation (4). LR is a complicated process involving the secretion of numerous cytokines and growth factors and the activation of metabolic networks (5). Although this process is histologically well described, the genes that orchestrate LR have only been partially characterized (6). Investigating the mechanisms involved in LR will offer new therapeutic strategies to rationally regulate and control hepatic function in a diseased state, which has caused this physiological process to gain increasing attention.

LR following PHx develops in three stages; the priming/initiation stage, the proliferation stage and the termination/inhibition stage $(7,8)$. A large number of growth-response genes are involved in these phases of LR. Furthermore, it is necessary to elucidate the complex interplay of numerous cellular events, particularly between the hepatocytes and liver sinusoidal endothelial cells (LSECs) (9). Angiogenesis is a fundamental requirement for organ development, function restoration and tumor growth. As a potent, diffusible and specific pro-angiogenic factor among several growth factors, vascular endothelial growth factor (VEGF) is important in liver regeneration, due in part to its effect on neovascularization (10). VEGF expression in the regenerating liver is mainly detected in the periportal hepatocytes, and promotes the proliferation of hepatocytes through the reconstruction of liver sinusoids by the proliferation of LSECs (11).

MicroRNAs (miRNAs or miRs) are a family of small non-coding RNAs that regulate target gene expression at the post-transcriptional level by blocking protein translation or inducing the degradation of gene messenger RNA (mRNA) after hybridization to its 3'-untranslated region (UTR) (12). miRNAs are capable of regulating every aspect of cellular activity, including differentiation and development, metabolism, proliferation, apoptotic cell death, viral infection and tumorigenesis (13). miRNAs are critical regulators of hepa- 
tocyte proliferation during LR (14). The response of the vascular endothelium to angiogenic stimuli is modulated by miRNAs. Thus, targeting the expression of miRNAs may be a novel therapeutic approach for diseases involving excess or insufficient vasculature (15). Hypoxia is a potent stimulant for angiogenesis, and hypoxia-inducible factor- $1 \alpha$ (HIF-1 $\alpha)$ is an essential transcriptional factor for adaptation against hypoxia (16). Many miRNAs also demonstrate dynamic changes in response to cellular stimulation, including exposure to hypoxia. Whether HIF-1 $\alpha$ is involved in the transcriptional regulation of miRNAs related to angiogenesis during LR, particularly miRNAs targeting VEGF, remains unknown.

In this study, we hypothesized that the expression of a specific miRNA in the regenerating liver may correlate closely with angiogenesis. To identify this miRNA, we screened seven candidate miRNAs, obtained from related databases, and correlated their expression with the VEGF-A gene. Furthermore, to investigate whether the expression of the selected miRNAs were able to be regulated by HIF-1 $\alpha$ mRNA, we established hypoxic conditions via the administration of cobalt chloride $\left(\mathrm{CoCl}_{2}\right)$ in isolated hepatocytes and evaluated the expression and association of HIF-1 $\alpha$ mRNA, the selected microRNA and VEGF-A mRNA under hypoxia.

\section{Materials and methods}

Bioinformatics analysis. miRNA target site prediction for the VEGF-A gene was performed using the starBase database (http://starbase.sysu.edu.cn) (17). StarBase links to five commonly used miRNA target prediction programs including TargetScan (www.targetscan.org), picTar (www.mdc-berlin.de/ en/research/research_teams/systems_biology_of_gene_regulatory_elements/projects/pictar), $\quad$ R N A 22 (http://cbcsrv.watson.ibm.com/rna22.html), PITA (http://genie.weizmann.ac.il/pubs/mir07/mir07_data.html) and miRanda (http://www.microrna.org/microrna/home.do), which provide a comprehensive search for intersections among the putative miRNAs predicted by the programs and target gene VEGF-A.

Animals and operative procedures. Specific pathogen-free eight-week-old male C57BL/C mice were purchased from the Experimental Animal Medicine Centre of Sichuan University (Chengdu, China). The mice were housed at room temperature and 55\% humidity and given access to food and water ad libitum throughout the experiment. After quarantine for one week, 12 mice, with a mean weight of $18.2 \pm 2.7 \mathrm{~g}$, were subjected to $70 \%$ PHx using the procedure described previously (18) under chloral hydrate $(50 \mathrm{mg} / \mathrm{kg}$, i.p.) anesthesia. Following surgery, the rats were further divided into three groups $(12,24$ and $48 \mathrm{~h}$ following PHx, $n=4 /$ group). At the indicated times, four mice were randomly sacrificed and the remnant regenerating liver tissues were collected and stored at $-80^{\circ} \mathrm{C}$. All experiments were approved by the Committee of the Experimental Use of Animals, Sichuan University, and performed in accordance with institutional animal care instructions approved by the Ethics Committee for Animals, Sichuan University.

Primary hepatocyte culture. Primary hepatocytes were obtained from mouse liver using in situ two-step collagenase liver perfusion (19) with minor modifications. The mean viability of hepatocytes was $90 \%$, as determined by trypan blue exclusion. The isolated hepatocytes were suspended in DMEM (Hyclone, Logan, UT, USA) supplemented with 10\% fetal calf serum (Invitrogen, Carlsbad, CA, USA), $1 \mathrm{mM}$ insulin and $1 \%$ penicillin/streptomycin (Sigma-Aldrich, St. Louis, MO, USA) and seeded in 6-well culture plates precoated with rat tail tendon collagen type I (Shengyou Biotech, Co., Ltd., Hangzhou, China) at $1 \times 10^{6}$ cells $/ \mathrm{ml}$. Subsequently, the primary hepatocytes were maintained for $4 \mathrm{~h}$ at $37^{\circ} \mathrm{C}$ in a $95 \% \mathrm{O}_{2}$ and $5 \% \mathrm{CO}_{2}$ atmosphere to allow for stabilization. Four hours later, the unattached cells were removed.

Hypoxia induction in primary hepatocytes. Hypoxia induction was achieved using DMEM medium containing HIF-1 $\alpha$-stabilizing agent $\mathrm{CoCl}_{2}$ (Sigma-Aldrich) at a final concentration of $100 \mu \mathrm{M}(20)$. In order to evaluate the gene expression levels, the cells were divided into three groups. In group $\mathrm{A}$, the hepatocytes were cultured without $\mathrm{CoCl}_{2}$ and used as a control. In groups $\mathrm{B}$ and $\mathrm{C}$, hepatocytes were cultured with $\mathrm{CoCl}_{2}$ for 12 and $48 \mathrm{~h}$, respectively.

miR-150 inhibitor transfection. Hepatocytes were seeded in 96-well plates at a density of $5 \times 10^{3}$ cells/well and transfected with micrOFFTM miR-150 inhibitor or a negative control (RiboBio Biotech., Co., Ltd., Guangzhou, China) at a final concentration of $100 \mathrm{nM}$ using Lipofectamine ${ }^{\mathrm{TM}} 2000$ (Invitrogen), according to the manufacturer's instructions. Total RNA and protein were extracted 24 and $48 \mathrm{~h}$ after transfection for quantitative PCR (qPCR) and western blot analysis.

qPCR for miRNA detection. Total RNA was extracted from prepared samples using TRIzol reagent (Invitrogen), according to the manufacturer's instructions. The quality and quantity of each RNA sample were measured spectrophotometrically using a NanoDrop 1000 (Thermo Scientific, Waltham, MA, USA) and the integrity of the RNA was assessed by $1 \%$ ethidium bromide agarose gel electrophoresis (Invitrogen). Equal amounts of $1 \mu \mathrm{g}$ RNA were reverse transcribed into cDNA using the miRcute miRNA first-strand cDNA synthesis kit (Tiangen Biotech Co., Ltd., Beijing, China), according to the manufacturer's instructions. Subsequently, qPCR was performed using the miRcute miRNA qPCR detection kit (Tiangen). The $20 \mu \mathrm{l}$ qPCR mixture consisted of $10 \mu \mathrm{l}$ $2 \mathrm{X}$ miRcute miRNA premix, $2 \mu \mathrm{l}$ forward primer $(2 \mu \mathrm{M}), 2 \mu \mathrm{l}$ reverse primer $(2 \mu \mathrm{M}), 1 \mu \mathrm{l}$ cDNA and $5 \mu \mathrm{l}$ RNase-free $\mathrm{ddH}_{2} \mathrm{O}$. The primers used are listed in Table I. PCR amplification was performed using a 1000-Series Thermal Cyclers real-time PCR detection system (Bio-Rad, Hercules, CA, USA) under the following conditions: $94^{\circ} \mathrm{C}$ for 2 min followed by 40 cycles of $94^{\circ} \mathrm{C}$ for $20 \mathrm{sec}, 62^{\circ} \mathrm{C}$ for $30 \mathrm{sec}$ and $72^{\circ} \mathrm{C}$ for $30 \mathrm{sec}$. The production of specific products was confirmed using melting curve analysis $\left(65-95^{\circ} \mathrm{C}\right)$ at the end of the amplification cycle. All experiments were performed in triplicate for each miRNA to obtain $\mathrm{Ct}$ values, and a non-template control was included on the same plate. A small stably expressed housekeeping RNA molecule, 5s rRNA (Rn5s), was included as an internal control to normalize gene expression levels and its primer product was purchased from Tiangen Biotech Co., Ltd. Data were 
analyzed using Bio-Rad CFX Manager software (Bio-Rad) and the relative expression (fold difference) of candidate genes (miR126-5p, 134, 150, 185, 29a, 29b and 29c) was calculated using the $2^{-\Delta \Delta \mathrm{Ct}}$ method.

qPCR for mRNA detection. Treated hepatocytes were harvested at the indicated times and total RNA was extracted using TRIzol reagent. RNA was reverse transcribed using the PrimeScript ${ }^{\circledR}$ RT reagent kit with gDNA Eraser (Takara, Dalian, China). cDNA was amplified by qPCR with a 1000-Series Thermal Cyclers real-time PCR detection system (Bio-Rad) and SYBR ${ }^{\circledR}$ Premix Ex Taq ${ }^{\mathrm{TM}}$ II (Tli RNaseH Plus) (Takara), according to the manufacturer's instructions. PCR was performed in a $10 \mu \mathrm{l}$ reaction mixture containing $5 \mu 1$ SYBR premix, $1 \mu 1$ forward primer and reverse primer, $1 \mu 1$ cDNA template and $3 \mu \mathrm{l}$ RNase-free $\mathrm{ddH}_{2} \mathrm{O}$. The housekeeping gene $\beta$-actin mRNA normalized the levels of cDNA. Primers for PCR are listed in Table I. The PCR conditions were as follows: HIF- $1 \alpha, 95^{\circ} \mathrm{C}$ for $3 \mathrm{~min}$ followed by 45 cycles of amplification at $95^{\circ} \mathrm{C}$ for $5 \mathrm{sec}$ and $57^{\circ} \mathrm{C}$ for $15 \mathrm{sec}$; VEGF-A, $95^{\circ} \mathrm{C}$ for $3 \mathrm{~min}$ followed by 40 cycles of amplification at $95^{\circ} \mathrm{C}$ for $10 \mathrm{sec}, 56^{\circ} \mathrm{C}$ for $10 \mathrm{sec}$ and $72^{\circ} \mathrm{C}$ for $10 \mathrm{sec}$; and $\beta$-actin, $95^{\circ} \mathrm{C}$ for $3 \mathrm{~min}$ followed by 40 cycles of amplification at $95^{\circ} \mathrm{C}$ for $5 \mathrm{sec}$ and $59^{\circ} \mathrm{C}$ for $10 \mathrm{sec}$. All the samples were amplified in triplicate and each experiment was repeated three times.

Western blot analysis. Liver tissues, or cultured hepatocytes, were homogenized and lysed in cell lysis buffer (Beyotime institute of Biotechnology, Nantong, China) containing phenylmethanesulfonyl fluoride (PMSF; Beyotime) for $30 \mathrm{~min}$ on ice. The lysates were centrifuged for $10 \mathrm{~min}$ at $10,612 \mathrm{x}$ g at $4^{\circ} \mathrm{C}$. Protein concentrations were determined using the BCA protein assay kit (Beyotime) with BSA as a standard. Equal amounts of protein were loaded onto a $10 \%$ SDS-polyacrylamide gel and separated intermittently for $1 \mathrm{~h}$ at $100 \mathrm{~V}$ on an electrophoresis system (Bio-Rad). Proteins were then transferred to a nitrocellulose membrane (Pall, Port Washington, NY, USA) for $2.5 \mathrm{~h}$ at $0.35 \mathrm{~mA}$. Membranes were blocked for $1 \mathrm{~h}$ with a blocking buffer (Beyotime) and subsequently incubated overnight at $4^{\circ} \mathrm{C}$ with a $1: 100$ dilution of primary rabbit anti-HIF-1 $\alpha$ or anti-VEGF polyclonal antibody (Boster Bioengineering Co., Ltd., Wuhan, China). A mouse anti-GAPDH polyclonal antibody (Boster) was used as a loading control to normalize protein levels. After washing four times with TBS-T for $15 \mathrm{~min}$, the immunoblots were incubated for $1 \mathrm{~h}$ with a 1:5,000 dilution of secondary horseradish peroxidase-conjugated goat anti-rabbit IgG (Boster), followed by detection with enhanced chemiluminescence (Beyotime) according to the manufacturer's instructions. Protein levels were visualized on the gel imaging and analysis system and quantified using the Quantity One 4.5 software (Bio-Rad). The gray ratio value between the target protein and GAPDH was used to calculate the relative expression level of target protein for the subsequent statistical analysis.

Statistical analysis. All data are expressed as the mean \pm SD. Statistical analysis was performed using one-way ANOVA and a Newman-Keuls test between groups using Statistical Package for the Social Science (SPSS) for Windows (SPSS, Inc., Chicago, IL, USA). Graphs were created with Origin 7.5
Table I. Primer sequences for qPCR.

\begin{tabular}{ll}
\hline Name & \multicolumn{1}{c}{ Sequence } \\
\hline miR-126-5p & 5'-GAGGCATTATTACTTTTGGTACG-3' \\
miR-134 & 5'-GTGACTGGTTGACCAGAGGG-3' \\
miR-150 & 5'-TCTCCCAACCCTTGTACCAGT-3' \\
miR-185 & 5'-TGGAGAGAAAGGCAGTTCCTG-3' \\
miR-29a & 5'-TAGCACCATCTGAAATCGGTTAA-3' \\
miR-29b & 5'-TAGCACCATTTGAAATCAGTGTTA-3' \\
miR-29c & 5'-GTAGCACCATTTGAAATCGGTTA-3' \\
HIF-1 $\alpha$ & F: 5'-ATCGCGGGGACCGATT-3' \\
& R: 5'-CGACGTTCAGAACTTATCTTTTTCTT-3' \\
VEGF-A & F: 5'-CTGTACCTCCACCATGCCAAGT-3' \\
& R: 5'-AGATGTCCACCAGGGTCTCAAT-3' \\
$\beta$-actin & F: 5'-AGAG GGAAATCGTGCGTGAC-3' \\
& R: 5'-CAATAGTGATGACCTGGCCGT-3' \\
\hline
\end{tabular}

qPCR, quantitative PCR; HIF- $1 \alpha$, hypoxia-inducible factor- $1 \alpha$; VEGF-A, vascular endothelial growth factor-A.

(OriginLab data analysis and graphing software, Northampton, MA, USA). $\mathrm{P}<0.05$ was considered to indicate a statistically significant difference.

\section{Results}

Computational predictions of miRNAs targeting VEGF-A. Since this study investigated the effect of miRNA profile change in the proliferating hepatocytes upon VEGF-A gene expression and aimed to identify an miRNA that was associated with the VEGF-A gene with relative specificity, we first analyzed target site intersections between mouse miRNAs and the VEGF-A gene using a prediction assay of miRNA target genes. Initially, in silico analysis predicted 22 putative miRNAs based on three reference sequences of the mouse VEGF-A gene (GenBank accession numbers: NM_009505, NM_001025257 and NM_001025250) using five bioinformatics algorithms (Table II). The animal miRNA target genes were roughly estimated since there was incomplete base pairing on binding sites between the miRNA and mRNA target sequences (21). In order to make full use of all the algorithm programs with different characteristics and to improve the accuracy rate of target prediction, we selected miRNAs that were simultaneously predicted by two or more of the five bioinformatics algorithms as putative regulators of VEGF-A. In total, 7 of the 22 putative miRNAs were selected for further screening in order to identify a specific miRNA with close correlation to the VEGF-A gene.

Screening of miRNAs targeting VEGF-A in the early phase of $L R$. Since PHx is considered to be the most potent reproducible stimulus for hepatocyte proliferation (22), we analyzed the liver tissues following $\mathrm{PHx}$ to explore changes in the expression of miRNAs in hepatocytes of the regenerating liver. We investigated which miRNA was regulated upon hypoxia in order to modulate the expression of VEGF-A in the 
A

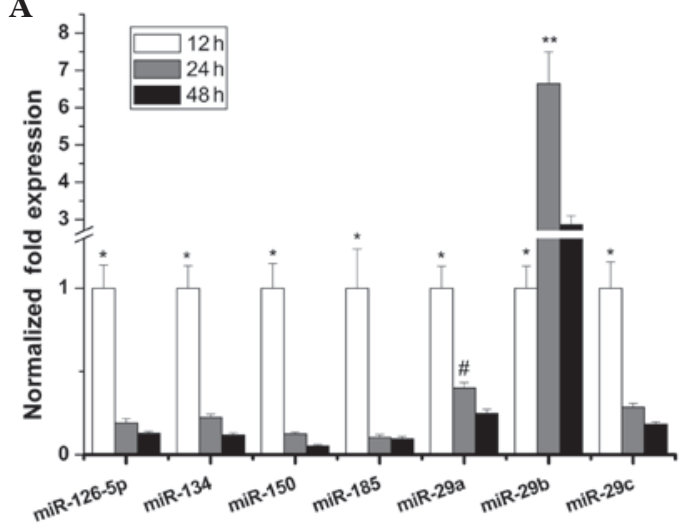

B

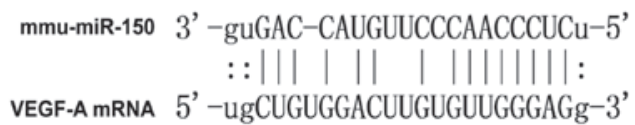

Figure 1. (A) Relative expression levels of seven miRNAs in the regenerating liver as determined by qPCR. Six of the seven candidate miRNAs, but not miR-29a, exhibited significant downregulation $>2$-fold between 12 to $24 \mathrm{~h}$ or $48 \mathrm{~h}$ following PHx ( $\mathrm{P}<0.01)$. miR-29a expression at $24 \mathrm{~h}$ following $\mathrm{PHx}$ was higher than that at $48 \mathrm{~h}$ following $\mathrm{PHx}\left({ }^{*} \mathrm{P}<0.05\right)$. There were significant differences in miR-29b expression levels at the three time-points $\left({ }^{* *} \mathrm{P}<0.01\right)$. miR-150 exhibited an $\sim 19$-fold reduction and the most prominent downtrend from 12 to $48 \mathrm{~h}$ following PHx. All results were normalized to $5 \mathrm{~s}$ rRNA expression. All data are presented as the mean \pm SD. (B) Incomplete base pairing between mmu-miR-150 and 3'-UTR of the VEGF-A gene miRNA, microRNA; qPCR, quantitative PCR; PHx, partial hepatectomy; UTR, untranslated region; VEGF-A, vascular endothelial growth factor-A.

seven miRNAs predicted to target VEGF-A. We measured the miRNA expression levels according to the variation tendency of VEGF-A expression in the early phase of LR. Alterations in the expression profiles of the miRNAs were examined using an miRNA microarray assay. However, the significantly altered miRNAs observed in the microarray results were selected for further validation by qPCR, as the microarray detects changes in gene expression without the need for high specificity and the microarray product of different sources may have inconsistent results. Thus, we directly detected substantial levels of seven miRNAs by qPCR instead of miRNA microarray assay and observed changes in their expression at three time-points $(12,24$ and $48 \mathrm{~h})$ once the total RNA was extracted from prepared liver samples. We identified that six of the miRNAs, with the exception of miR-29b, were downregulated $>2$-fold between 12 and $48 \mathrm{~h}$ (Fig. 1A). Among them, miR-150 was downregulated $\sim 19$-fold from $12-48 \mathrm{~h}$ and demonstrated the most marked downwards trend. The direct binding site of miR-150 to the VEGF target sequence is shown in Fig. 1B.

Effect of miR-150 inhibitors on VEGF-A gene expression. miRNAs with significant alterations in their expression levels, as determined by the microarray results, were generally considered to correlate closely with different physiological or pathological phenomena. We infer that the increased production of VEGF in the early stage of LR is due to a global downregulation in the production of certain miRNAs. Of all the miRNAs predicted to target VEGF-A, miR-150 was selected for further investigation as it was the most downregulated. In view of an interaction between miR-150 and VEGF-A

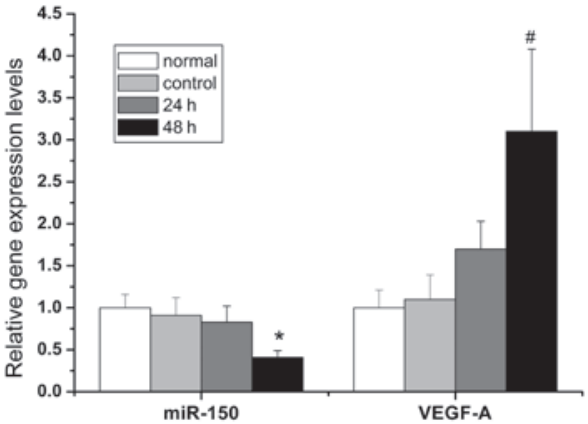

Figure 2. Effect of miR-150 inhibitor on miR-150 and VEGF-A gene expression in primary hepatocytes. qPCR detected the expression levels of the miR-150 and VEGF-A genes in the hepatocytes. Changes in the expression levels of the miR-150 and VEGF-A genes were not identified to be significantly different among the normal control group, the negative control group and the group assessed $24 \mathrm{~h}$ after treatment with the miR-150 inhibitor. At $48 \mathrm{~h}$ after tranfection, miR-150 gene expression declined 2.4 -fold $\left({ }^{*} \mathrm{P}<0.05\right)$ with VEGF-A gene expression increasing 3.1-fold $\left({ }^{\#} \mathrm{P}<0.05\right)$ compared with the normal control cells. 5s rRNA and $\beta$-actin mRNA were used as internal controls for miR-150 and VEGF-A gene expression, respectively. The data are presented as the mean \pm SD for three independent experiments. miR, microRNA; VEGF-A, vascular endothelial growth factor-A; qPCR, quantitative PCR.

mRNA with the highest degree of correlation, we evaluated the role of miR-150-mediated regulation of VEGF-A gene expression. The miR-150 inhibitors and a negative control were transfected into the hepatocytes for 24 and $48 \mathrm{~h}$. As a chemosynthetic and modified inhibitor, the micrOFF miRNA inhibitor specifically bound the mature target miRNA and suppressed its expression. The efficacy of downregulating the expression of the miR-150 gene and upregulating the expression of the VEGF-A gene was examined by qPCR and western blot analysis. Fig. 2 demonstrates that the miR-150 inhibitors effectively suppressed the expression of miR-150 2.4-fold $48 \mathrm{~h}$ after transfection and promoted the corresponding transcription and translation of the VEGF-A gene. The mRNA and protein levels of VEGF-A increased 3.1-fold and 2.6-fold, respectively, $48 \mathrm{~h}$ after transfection with the miR-150 inhibitors compared with the control group. The differences were not statistically significant at $24 \mathrm{~h}$ when compared with the control group (Fig. 3).

$H I F-1 \alpha$ protein expression in the early phase of $L R$. In order to evaluate the hypoxic conditions in the early phase of LR, we selected the HIF-1 $\alpha$ protein as a detection index to indicate the degree of hypoxia. Intraoperatively excised liver tissues served as the normal control $(0 \mathrm{~h})$. Western blot analysis demonstrated that HIF-1 $\alpha$ protein expression levels were increased $12 \mathrm{~h}$ after PHx and continued increasing until the highest expression level was achieved $24 \mathrm{~h}$ after PHx with an $~ 4$-fold increase relative to the control group (Fig. 4).

HIF-1 $\alpha$, miR-150 and VEGF-A mRNA expression in hypoxia-induced hepatocytes. To determine whether miR-150 was regulated by hypoxia, primary hepatocytes were isolated and hypoxia was induced with $\mathrm{CoCl}_{2}$. As shown in Fig. 5, the expression of HIF-1 $\alpha$ mRNA was low in the normoxic state, but increased at $12 \mathrm{~h}$ (2.7-fold vs. control) and increased further at $48 \mathrm{~h}$ (4-fold vs. control) following the administration 

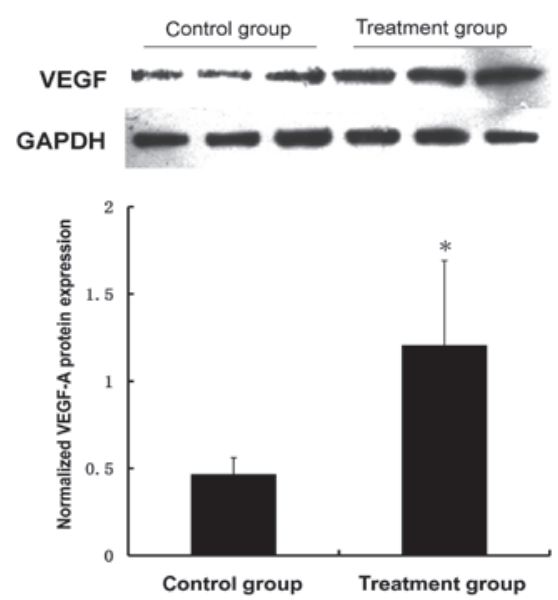

Figure 3. Effect of the miR-150 inhibitor on VEGF-A protein expression in primary hepatocytes. The fold-change of protein expression levels was calculated by gray scanning the western blotting bands. At $48 \mathrm{~h}$ following transfection, the expression of VEGF-A protein increased 2.6-fold ( $\mathrm{P}<0.05)$ in the group treated with miR-150 inhibitor compared with that treated with a negative control. The images are representative of three independent experiments. GAPDH was used as a loading control. The data are presented as the mean \pm SD. VEGF-A, vascular endothelial growth factor-A; miR, microRNA.

$\mathbf{A}$

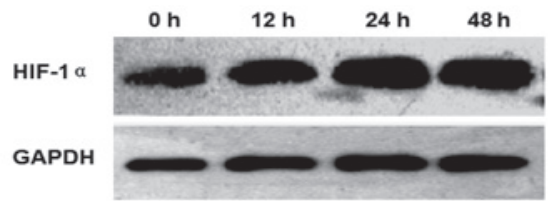

B

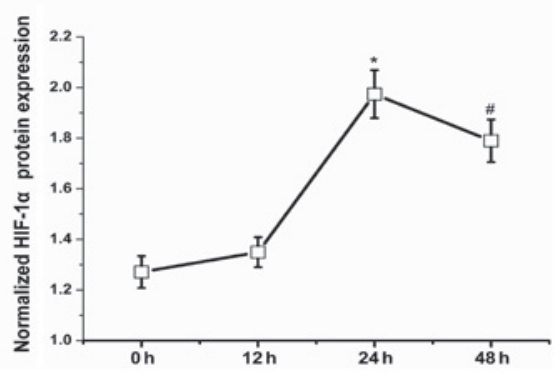

Figure 4. (A) Western blot analysis of HIF-1 $\alpha$ protein levels and (B) densitometric results of western blot bands. The expression levels of HIF-1a protein increased $24 \mathrm{~h}$ after $\mathrm{PHx}$, as demonstrated in (A), and the densitometry results shown in (B) are consistent with the bands. The images are representative of three independent experiments. GAPDH was used as a loading control. The data are presented as the mean $\pm \mathrm{SD},{ }^{*} \mathrm{P}<0.01$ vs. $0 \mathrm{~h}$ and $12 \mathrm{~h} ;{ }^{*} \mathrm{P}<0.05$ vs. $24 \mathrm{~h}$. HIF-1 $\alpha$, hypoxia-inducible factor- $1 \alpha$; PHx, partial hepatectomy.

of $\mathrm{CoCl}_{2}$. Moreover, the expression of miR-150 declined and VEGF-A mRNA increased $48 \mathrm{~h}$ after hypoxia with differences of 4.2-fold and 3.1-fold, respectively, relative to the normal control. However, the expression of miR-150 and VEGF-A mRNA was not significantly different from the control $12 \mathrm{~h}$ after hypoxia.

\section{Discussion}

In the early phase of LR, proliferating hepatocytes show hypoxia-induced VEGF expression, which initiates a process that aims to achieve the proper flow of blood through the

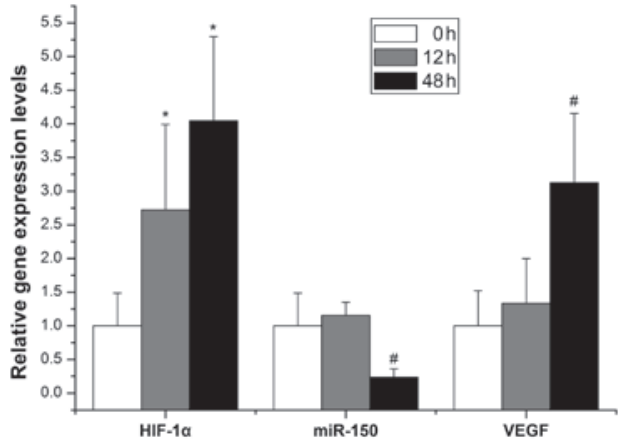

Figure 5. Evolution of miR-150, HIF-1 $\alpha$ mRNA and VEGF-A mRNA levels in primary hepatocytes treated with $\mathrm{CoCl}_{2}$ at the indicated time points $(0,12$ and $48 \mathrm{~h}$, respectively), as determined by qPCR. A gradual increase in HIF-1 $\alpha$ mRNA expression was observed with increasing time (2.7-fold at $12 \mathrm{~h}$ and 4 -fold at $48 \mathrm{~h}$ vs. control, ${ }^{*} \mathrm{P}<0.05$ ). By contrast, elevated HIF-1o mRNA levels resulted in a decrease in miR-150 expression and an increase in VEGF-A mRNA expression at $48 \mathrm{~h}$ compared with the control $\left({ }^{\sharp} \mathrm{P}<0.05\right)$. All results were normalized to 5s rRNA or $\beta$-actin mRNA expression. All_data are presented as the mean \pm SD of three independent experiments $(n=3$ per condition). HIF-1 $\alpha$, hypoxia-inducible factor- $1 \alpha$; VEGF-A, vascular endothelial growth factor-A; qPCR, quantitative PCR; mRNA, messenger RNA; miR, microRNA.

liver (23). However, changes in the miRNA profile that promote the expression of the VEGF gene upon hypoxia remain unknown. In order to determine these changes, the present study focused on miRNAs targeting VEGF-A. Initially, we examined the dynamic change of miRNAs predicted to target VEGF-A in the early phase of LR and selected miR-150 as the miRNA with the highest potential to modulate VEGF-A gene expression. Additionally, we observed the effect of miR-150 knockdown on VEGF-A gene expression in primary hepatocytes. Finally, we demonstrated the kinetics of HIF-1 $\alpha$ expression in mouse regenerating liver and the hypoxia responsiveness of miR-150 by assessing HIF- $1 \alpha$ and VEGF-A gene expression upon hypoxic exposure.

miRNAs have multiple gene targets and each target may be regulated by multiple miRNAs (24). Thus, we performed computational predictions with five algorithms in order to guarantee the accuracy rate. Based on the computer analysis of putative binding sites on the target gene, the interactions of the investigated miRNAs with VEGF are purely speculative. In principle, miRNAs are hypothesized to negatively regulate target gene expression by inhibiting their stability or translation. Thus, the expression of the target gene is inversely proportional to its upstream miRNAs if their regulating relationship is highly specific. A previous report demonstrated that VEGF was detected as early as $12 \mathrm{~h}$ following PHx, peaked at $48-72 \mathrm{~h}$ and gradually declined to the baseline level after 8-10 days (25). Our results suggest that miR-150 exhibited the most marked decrease from 12-48 $\mathrm{h}$ following PHx among the seven miRNAs predicted to target VEGF-A. Furthermore, we revealed that the expression of VEGF-A mRNA was negatively correlated with miR-150 expression in hepatocytes $48 \mathrm{~h}$ after exposure to hypoxia. These results suggest that there is an interaction between miR-150 and VEGF-A mRNA for their inverse co-expression with a high degree of correlation. Shen et al (26) observed a decrease in the levels of miR-150 in the ischemia-induced retinal neovascularization model compared with normal retinas, and the luciferase levels of a VEGF reporter were downregulated by 
co-transfection with pre-miR-150. In the present study, our results demonstrated that the knockdown of miR-150 using the micrOFF miR-150 inhibitor promoted the expression of VEGF-A mRNA and protein in primary hepatocytes. Evidence from the validation of gene structure and function demonstrates that VEGF-A may be a target of miR-150 and miR-150 may be crucial in the regulation of VEGF-A gene expression and angiogenesis during mouse LR.

Following cell division in the initial stage of LR, hepatocytes are found in clusters and no longer associate with sinusoids. Ninomiya et al (27) hypothesized that the abrupt regenerative response of hepatocytes to resection suppresses sinusoids, resulting in local hypoxia and induction of the HIF-1 $\alpha$ genes in the regenerating hepatocytes. In other words, hypoxia occurs due to the incongruous replication of hepatocytes and LSECs. Our results demonstrated that expression of the HIF-1 $\alpha$ protein in mouse tissues in LR increased and peaked at $24 \mathrm{~h}$, similar to the result observed by Maeno et al (28). We also revealed that HIF-1 $\alpha$ expression increased in primary hepatocytes following the administration of $\mathrm{CoCl}_{2}$, which indicates that a hypoxia-induced hepatocyte model was established. This is due to the fact that cobalt has been widely used to mimic a hypoxic response and induce HIF-1 $\alpha$ production in cultured cells (29). Although no marked change in miR-150 and VEGF-A mRNA expression was observed $12 \mathrm{~h}$ after hypoxia treatment in hypoxia-induced hepatocytes when HIF-1 $\alpha$ mRNA levels had already started to increase, we assume that HIF-1 $\alpha$ mRNA had not yet translocated from the cytoplasm into the nucleus where HIF-1 $\alpha$ functions in combination with its target gene. These results suggested that miR-150 may be identified as a downregulated miRNA by hypoxia, not only during LR, but also in the anoxic model of primary hepatocytes, since its production is dependent on low levels of HIF-1 $\alpha$ expression. Thus, we conclude that HIF-1 $\alpha$ participates in the transcriptional regulation of miR-150.

Previous studies have focused on the role of VEGF in LR. However, little is known with regards to the underlying molecular mechanisms by which the VEGF gene is produced and modulated. The discovery of miRNAs offers a unique insight into their ability to regulate target genes.

In conclusion, we provide evidence that the expression of miR-150 is specifically related to VEGF-A and is subject to negative regulation by HIF- $1 \alpha$ during the early phase of LR. Therefore, miR-150 serves as an indirect bridge between HIF-1 $\alpha$ and VEGF-A, suggesting that there is an alternative pathway for the HIF- $1 \alpha$ regulation of VEGF-A. The phenomenon identified in this study will extend our knowledge of factors controlling liver regeneration.

\section{Acknowledgements}

This work was supported by grants from the Sichuan provincial science and technology support program (No.2010FZ0016).

\section{References}

1. Michalopoulos GK: Liver regeneration: alternative epithelial pathways. Int J Biochem Cell Biol 43: 173-179, 2011.

2. Donahower B, McCullough SS, Kurten R, et al: Vascular endothelial growth factor and hepatocyte regeneration in acetaminophen toxicity. Am J Physiol Gastrointest Liver Physiol 291: G102-G109, 2006.
3. Michalopoulos GK: Liver regeneration after partial hepatectomy: critical analysis of mechanistic dilemmas. Am J Pathol 176: 2-13, 2010.

4. Hua ZY, Song J, Cheng F, et al: The effect of hepatocyte growth factor on the initiation phase of liver regeneration after cold ischemia in a rat model of small-for-size liver transplantation. Hepatogastroenterology 59: 1548-1552, 2012.

5. Fausto N, Campbell JS and Riehle KJ: Liver regeneration. Hepatology 43: S45-S53, 2006.

6. Böhm F, Köhler UA, Speicher T and Werner S: Regulation of liver regeneration by growth factors and cytokines. EMBO Mol Med 2: 294-305, 2010.

7. Chen JA, Shi M, Li JQ and Qian CN. Angiogenesis: multiple masks in hepatocellular carcinoma and liver regeneration. Hepatol Int 4: 537-547, 2010.

8. Tarlá MR, Ramalho FS, Ramalho LN, et al: A molecular view of liver regeneration. Acta Cir Bras 21 (Suppl 1): 58-62, 2006.

9. Crawford SE: Vascular interference: a blockade to tumor epithelial growth. Hepatology 39: 1491-1494, 2004.

10. Bockhorn M, Goralski M, Prokofiev D, et al: VEGF is important for early liver regeneration after partial hepatectomy. J Surg Res 138: 291-299, 2007.

11. Taniguchi E, Sakisaka S, Matsuo K, Tanikawa K and Sata M: Expression and role of vascular endothelial growth factor in liver regeneration after partial hepatectomy in rats. J Histochem Cytochem 49: 121-130, 2001.

12. Bartel DP: MicroRNAs: genomics, biogenesis, mechanism, and function. Cell 116: 281-297, 2004.

13. Chen XM: MicroRNA signatures in liver diseases. World J Gastroenterol 15: 1665-1672, 2009.

14. Song G, Sharma AD, Roll GR, et al: MicroRNAs control hepatocyte proliferation during liver regeneration. Hepatology 51 : 1735-1743, 2010.

15. Fish JE and Srivastava D: MicroRNAs: opening a new vein in angiogenesis research. Sci Signal 2: pe1, 2009.

16. Chaudhuri S, McCullough SS, Hennings L, et al: Acetaminophen hepatotoxicity and HIF-1 $\alpha$ induction in acetaminophen toxicity in mice occurs without hypoxia. Toxicol Appl Pharmacol 252: 211-220, 2011.

17. Yang JH, Li JH, Shao P, et al: starBase: a database for exploring microRNA-mRNA interaction maps from Argonaute CLIP-Seq and Degradome-Seq data. Nucleic Acids Res 39: D202-D209, 2011.

18. Mitchell C and Willenbring $\mathrm{H}$ : A reproducible and well-tolerated method for $2 / 3$ partial hepatectomy in mice. Nat Protoc 3: 1167-1170, 2008.

19. Li WC, Ralphs KL and Tosh D: Isolation and culture of adult mouse hepatocytes. Methods Mol Biol 633: 185-196, 2010.

20. Fradette $\mathrm{C}$ and du Souich P: Hypoxia-inducible factor-1 and activator protein-1 modulate the upregulation of CYP3A6 induced by hypoxia. Br J Pharmacol 140: 1146-1154, 2003.

21. Yu CH, Xu CF and Li YM: Association of MicroRNA-223 expression with hepatic ischemia/reperfusion injury in mice. Dig Dis Sci 54: 2362-2366, 2009.

22. Yao XM, Zhao J, Li Y and Li Y: Effects of bicyclol on liver regeneration after partial hepatectomy in rats. Dig Dis Sci 54: 774-781, 2009.

23. Kajdaniuk D, Marek B, Foltyn W and Kos-Kudła B: Vascular endothelial growth factor (VEGF) - part 1: in physiology and pathophysiology. Endokrynol Pol 62: 444-455, 2011.

24. Lewis BP, Shih IH, Jones-Rhoades MW, Bartel DP and Burge CB: Prediction of mammalian microRNA targets. Cell 115: 787-798, 2003.

25. Mohammed FF and Khokha R: Thinking outside the cell: proteases regulate hepatocyte division. Trends Cell Biol 15: $555-563,2005$.

26. Shen J, Yang X, Xie B, et al: MicroRNAs regulates ocular neovascularization. Mol Ther 16: 1208-1216, 2008.

27. Ninomiya M, Shirabe K, Terashi T, et al: Deceleration of regenerative response improves the outcome of rat with massive hepatectomy. Am J Transplant 10: 1580-1587, 2010.

28. Maeno H, Ono T, Dhar DK, et al: Expression of hypoxia inducible factor-1alpha during liver regeneration induced by partial hepatectomy in rats. Liver Int 25: 1002-1009, 2005.

29. Battaglia V, Compagnone A, Bandino A, et al: Cobalt induces oxidative stress in isolated liver mitochondria responsible for permeability transition and intrinsic apoptosis in hepatocyte primary cultures. Int J Biochem Cell Biol 41: 586-594, 2009. 